\title{
AUTOMOTIVE IVHM: TOWARDS INTELLIGENT PERSONALISED SYSTEMS HEALTHCARE
}

\author{
Campean, Felician (1); Neagu, Daniel (1); Doikin, Aleksandr (1); Soleimani, Morteza (1); Byrne, \\ Thomas (1); Sherratt, Andrew (2) \\ 1: University of Bradford, Automotive Research Centre; 2: Jaguar Land Rover
}

\begin{abstract}
Underpinned by a contemporary view of automotive systems as cyber-physical systems, characterised by progressively open architectures increasingly defined by their interaction with the users and the smart environment, this paper provides a critical and up-to-date review of automotive Integrated Vehicle Health Management (IVHM) systems. The paper discusses the challenges with prognostics and intelligent health management of automotive systems, and proposes a high-level framework, referred to as the Automotive Healthcare Analytic Factory, to systematically collect and process heterogeneous data from across the product lifecycle, towards actionable insight for personalised healthcare of systems.
\end{abstract}

Keywords: Large-scale engineering systems, Digital / Digitised engineering value chains, Product Lifecycle Management (PLM), IVHM, Digital twin

\section{Contact:}

Campean, Felician

University of Bradford

School of Engineering

United Kingdom

F.Campean@bradford.ac.uk 


\section{INTRODUCTION}

\subsection{From cars to automotive systems of systems}

Road vehicles have seen a significant transformation over the past 50 years, substantially influenced by technology developments in electronics, software and networks, as illustrated in Figure 1. While in the 1970s automotive innovation was predominantly in the electro-mechanical domain, by 1990s embedded mechatronic systems have become common in the vehicle architecture, with innovation focussed on enhancing environmental and safety performance of the vehicle. By the 2000s the innovation focus had moved towards enhancing the users' experience of the interaction with vehicle as a system, by providing enhanced, intelligent control features for the navigation task (through Advanced Driver Assistance Systems / features, ADAS) as well as the vehicle environment. Nowadays, inter-connectivity of vehicles plays a prominent role in automotive innovation, enabling integration of the vehicle with many other systems. This increased integration with the environment, underpinned by proliferation of IoT technology, means that the vehicle can be regarded as a cyber-physical system increasingly defined by its interaction with multiple systems of systems (SoS). This offers some important opportunities for an automotive Product Development (PD) organisation, as all vehicles will be part of an extended and open PD SoS, thus enabling continuous communication with the vehicles to operate vehicle updates and intelligent health management based on remote diagnostics and contextualised usage information. Integration of vehicles within other smart SoS (e.g. smart traffic, smart city) provide enhanced opportunities to innovate with features that provide further utility to the user and/or the environment, facilitating the servitisation agenda prevalent in the emerging business models. However, this leads to an exponential increase in the interactions of the vehicle with other systems in multiple smart ecosystems, with many of these interactions having an open non-deterministic nature. This increases the amount of uncertainty that needs to be handled throughout the product lifecycle, with the most significant impact for the design stage, early in the product development phases.

Figure 1 also illustrates the vehicle as a complex system, within a system of systems context, defined across a number of levels or scales. A vehicle is made up of several thousand physical parts (components, assemblies, subsystems), which interact dynamically during the use of the vehicle to deliver the functional goals of the system. Most of the components and subsystems in a modern vehicle have a composite structure, as combination of physical (electromechanical) systems with smart actuators that embed sensors and software control structures. The increase in complexity is also reflected by the ubiquitous engineering trans-disciplinarity of automotive product development: automotive engineering is no longer a combination of mechanical and electrical engineering, as it now includes an increasingly broad range of disciplines and expertise ranging from electronics and control engineering, to software engineering, networks and communications engineering, as well as IoT, artificial intelligence and data science. Therefore, the automotive product development organisation is also characterised by multi-disciplinary heterogeneity just like the automotive systems themselves.

\subsection{SoS perspective on automotive IVHM systems}

Integrated Vehicle Health Management (IVHM) refers to the unified capability of a system of systems to assess current or future state of a member system health, and to integrate this within a framework of available resources and operational demand (Wilmering, 2017). The recently introduced aerospace and automotive recommended practice JA6268 (SAE International, 2018), provides a useful six-point reference scale to define a prescriptive vehicle health capability guide, summarised in Table 1. This IVHM framework maps the capability scale from reactive maintenance based entirely on human led diagnostics (level 0); to increased levels of diagnostics support from automatic diagnostics (or diagnostic trouble codes (DTC) originally introduced to support compliance with emissions legislation, see Holland, 2010); and remote health monitoring enabled by the vehicle connectivity to the environment, enabling predictive maintenance action via further analysis of the remote diagnostics information. A major transition in the IVHM capability scale occurs between levels 2 and 3, where prognostics and predictive analytics are employed to implement condition based preventive and prescriptive maintenance actions, including the necessary, associated logistics. Levels 3, 4 and 5 are underpinned by the systems of systems technologies like IoT. Levels 4 and 5 mark the move from component based healthcare to integrated vehicle level health 
management, which brings further reliance on artificial intelligence driven predictive control deeply integrated within the SoS context.

Figure 1 also illustrates the alignment of the IVHM capability with the evolution of the automotive systems in time and in size, scale and SoS integration. JA2628 also provides a comprehensive guideline for system analysis to integrate component design data in a system IVHM (Felke et al., 2017).

Digital twin, a visionary concept introduced with the NASA's Apollo programs (Rosen et al., 2015), proposes a lifecycle based simulation approach to support the advanced IVHM capability. Digital twin research has received a significant attention in recent years, aiming to deliver both conceptual and technology developments to support data driven decision making within an Industry 4.0 SoS ecosystem (Rosen et al., 2015; Weier et al., 2016; Schleich et al., 2017; Negri et al., 2017, Kritzinger et al., 2018). The potential of the lifecycle approach to support enhanced vehicle system healthcare is an approach that will be considered in this paper

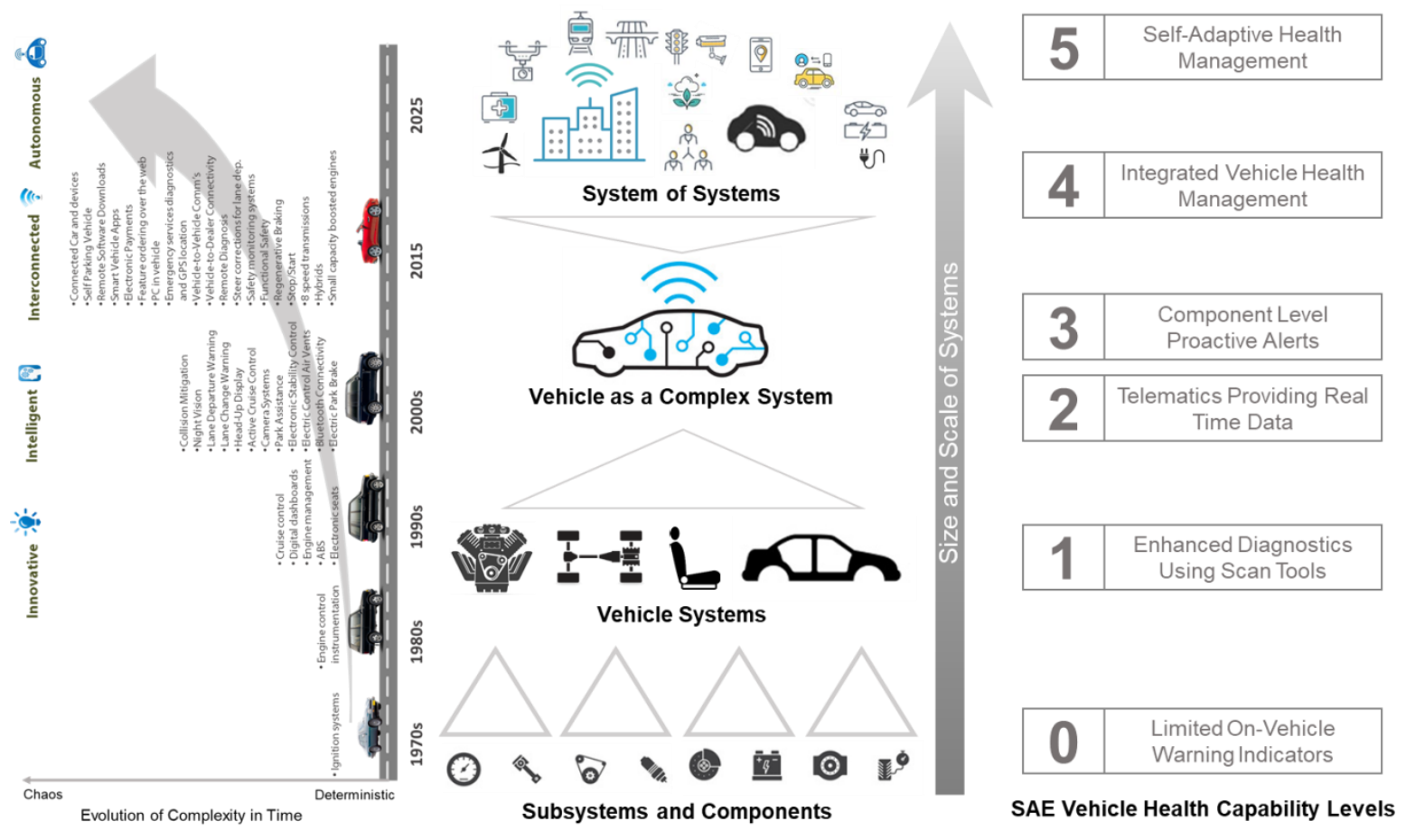

Figure 1. Characteristics of automotive cyber-physical systems

Table 1. SAE IVHM capability levels

\begin{tabular}{|c|l|l|}
\hline Level & $\begin{array}{l}\text { Vehicle Health } \\
\text { Capability }\end{array}$ & Description \\
\hline 0 & $\begin{array}{l}\text { Limited On-Vehicle } \\
\text { Warning Indicators }\end{array}$ & $\begin{array}{l}\text { Service actions prompted by scheduled maintenance intervals or } \\
\text { when the vehicle operator is alerted by malfunction indicator } \\
\text { lights, simple gauges or observes and reports a performance issue. }\end{array}$ \\
\hline 1 & $\begin{array}{l}\text { Enhanced Diagnostics } \\
\text { using Portable } \\
\text { Maintenance Aids }\end{array}$ & $\begin{array}{l}\text { On-vehicle diagnostic insight using automatics scanners to extract } \\
\text { vehicle operating parameters and diagnostic codes calculated and } \\
\text { retained on-board specifically to enhance the diagnostic process. }\end{array}$ \\
\hline 2 & $\begin{array}{l}\text { Remote Health } \\
\text { Monitoring }\end{array}$ & $\begin{array}{l}\text { Real time vehicle diagnostic data available via telematics / remote } \\
\text { data link; Data used to monitor / analyse real-time performance. }\end{array}$ \\
\hline 3 & $\begin{array}{l}\text { Component Level } \\
\text { Proactive Alerts }\end{array}$ & $\begin{array}{l}\text { On-line component health status available with severity indication } \\
\text { (R/A/G) facilitating limited condition based maintenance. }\end{array}$ \\
\hline 4 & $\begin{array}{l}\text { Vehicle Level Health } \\
\text { Management }\end{array}$ & $\begin{array}{l}\text { On-line cross-system or vehicle-level health indicators available } \\
\text { along with estimated prognostics for critical function performance } \\
\text { and recommended actions for condition based maintenance. }\end{array}$ \\
\hline 5 & $\begin{array}{l}\text { Self-adaptive Health } \\
\text { Management }\end{array}$ & $\begin{array}{l}\text { System has capability of autonomous, real-time, self-adaptive } \\
\text { control and optimization to extend vehicle operation and enhance } \\
\text { mission completion / safety, given system diagnostic. }\end{array}$ \\
\hline
\end{tabular}




\subsection{Research vision: intelligent, personalised powertrain healthcare}

The work in this paper is carried out from the perspective of an automotive powertrain system, treated as a CPS within a SoS context, and aims to explore challenges and opportunities towards establishing a framework for Intelligent, Personalised Powertrain Healthcare (IPPHC).

\subsubsection{Motivating case study scenario}

The case for IPPHC will be illustrated on the basis of a powertrain subsystem, i.e. the exhaust afterareatment $(\mathrm{A} / \mathrm{T})$. The exhaust aftertreatment system has the function of treating the engine out gaseous emissions to ensure that tailpipe discharged gases meet the environmental legislation - in terms of emissions content (NOx and particulates), temperature and noise.

Arguably, the aftertreatment system can be regarded as a complex multi-disciplinary system in its own right: it integrates electromechanical components with chemical reactors, with multiple sensors and software to monitor and control the performance of the system. The multi-physics nature of the A/T system yields a significant internal complexity of the system (in terms of complexity of the interactions between the thermal and chemical processes), which also influence and are influenced by air flow and thermal energy functional devices (such as turbochargers and heat / electrical energy converters that are often part of the aftertreatment system technology mix). For some current applications, the aftertreatment system is connected in real time to the cloud for performance monitoring and control.

A current challenge for the automotive powertrain systems is to evolve towards meeting the emerging demands of the shift in legislation towards "real world driving emissions" - which means that legislated tailpipe emissions have to be met on actual journeys rather than standardised reference journeys tested under lab conditions. As a scenario for the (near) future, it can be envisaged that the level of allowed tailpipe emissions will increasingly depend on the state of the environmental variables. For example, in a smart mega city, the level of allowable traffic emissions could be dynamically and differentially set in relation to current location and environmental conditions. The allowable target cascaded to an individual vehicle will consequently be a function of the volume of traffic. The aftertreatment control system will have to communicate with the smart environment to pick up the current level of legislated emissions, and to adjust the control of the plant in relation to the current system conditions (vehicle state; propulsion system state - e.g. state of charge of the battery for the electric propulsion; driving route, and driver behaviour), to ensure that vehicle meets the tailpipe emissions target. From the point of view of the smart SOS (i.e. the smart city) it would also be expected that the powertrain system should be able to self-certify against the legislated target and communicate this to the Smart City.

In order to deliver to this challenge, the aftertreatment system will have to demonstrate robust performance against the following properties:

1. Self-awareness - relating to the self-understanding the current state of the physical system and its ability to perform the required functions with predictable level of confidence;

2. Environmental awareness - denoting the ability of the system to robustly communicate with its environment, and to analyse the environmental data to gather the information needed for optimal/ smart predictive control, such that predictive performance can be achieved even over transient conditions;

3. Self-certification and assurance properties of the system - whereby the system can confirm with given confidence that the system will be meeting the service requirements for the next time window, or the duration of the mission (e.g. over the current intended journey);

4. System health management - capability of the system to provide predictive and pro-active control to ensure self-health management of the system in relation to its mission requirement.

\subsubsection{Objectives and outline}

The objective of this paper is to provide a systematic review of challenges with prognostics and healthcare for automotive systems, and to discuss the key research challenges. The challenges will be contextualised with reference to automotive powertrain systems undertaken by the authors. In particular the challenges will be discussed in relation to IVHM implementations in other fields, in particular aerospace. The paper proposes a high-level framework, referred to as the Automotive Healthcare Analytics Factory, for a lifecycle approach to prognostics and healthcare, coherent with the Industry 4.0 digital twin concepts, outlining the key research challenges associated with the framework development. 


\section{REVIEW OF PROGNOSTICS AND IVHM CHALLENGES}

\subsection{Diagnostics, prognostics and health management}

Historically, both diagnostics and prognostics originate from the medical field. Diagnostics analysis relates to the investigation of the cause or nature of a condition, situation, or problem associated with the current state of a system. Prognostics on the other hand is concerned with predicting the future (either end of life or remaining useful life, RUL) based on the analysis of the available pertinent data. From an engineered system point of view diagnostics is the process of detecting and identifying a fault or failure mode of a system (fault identification) and the analysis of causal chains leading to the identification of the most likely root cause (fault isolation or problem location). Conventional diagnostics analysis is conducted when a fault has already occurred, and is therefore a reactive process for a maintenance decision, and thus cannot prevent downtime and corresponding expenses. Predictive diagnostics systems continuously monitor the state of the system to identify and flag up fault states as soon as they occur, and thus reduce the repair downtime and costs.

Prognostics employ prediction techniques that, based on the evaluation of the current condition of a component and conditional on future load and environmental exposure, provide an estimate of the end of life expressed in relation to the lifetime variable (time, cycles, miles, events), when the component or subsystem will no longer operate within its stated functional specifications. Prognostics are generally associated with physical components and subsystems that are subject to degradation mechanisms that can be described or modelled by continuous hazard functions of the load cycles experienced by the component. A life prediction model for the component can be generally derived through linear damage accumulation models, even if the system does not exhibit an immediately measurable damage characteristic. In practice, RUL is the most common prognostics metric, used to extract information on the likelihood of failure within a finite time horizon, based on the current inferred condition and conditional on the forecasted usage within the prediction timeframe.

Health management is the process of taking timely, appropriate maintenance intervention action based on the information generated from diagnostics and prognostics analysis (Lee et al., 2014). It focusses on assessing the impact of failure to implement optimal maintenance actions to minimise impact of loss onto the system and the user. Figure 2 illustrates the diagnostics and prognostics driven health management (Kim et al., 2017).

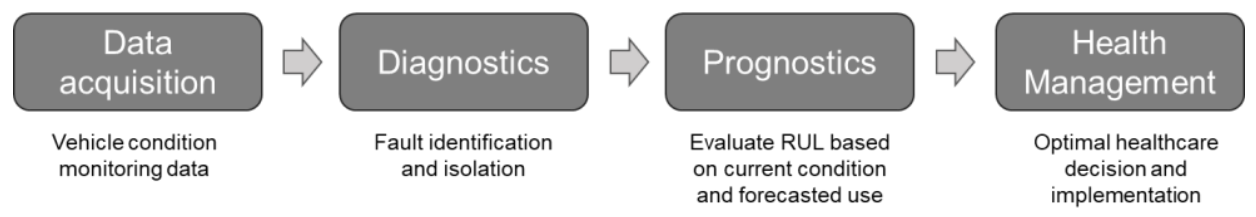

Figure 2. Prognostics and health management process flow

\subsection{Review of prognostics algorithms}

In general, prognostics methods can be categorised into phenomenological or model based (usually underpinned by modelling the physics of failure) and behavioural or data driven (Ellatar et al., 2016).

The model based approach to prognostics is illustrated in Figure 3, highlighting some of the uncertainties associated with RUL estimation. Degradation modelling against a known fault or mechanism is underpinned by observations (either direct or indirect) of the degradation variable at successive lifetime (measured in units of time, mileage, cycles, etc) intervals $t_{i}, . ., t_{n}$, to establish a model for degradation (as damage accumulation). Wei et al. (2017) presented a unified framework for life prediction modelling based on damage accumulation for automotive components, based on life test data. It is important to note that damage is also a function of the level of load or stress $(S)$ applied on the component. Underpinned by the life prediction model, RUL provides an estimate of the expected remaining life (measured in the units of the lifetime variable), based on an assumption about the future duty cycle that will be seen by the component. As an associate measure of prognostics, it is also of interest to evaluate the probability of failure (or failure free operation) over a defined time horizon $t_{H}$. This probability also depends whether the ultimate failure criteria is defined in relation to a threshold, or whether failure is itself a random variable of the damage, as illustrated in Figure 3. 


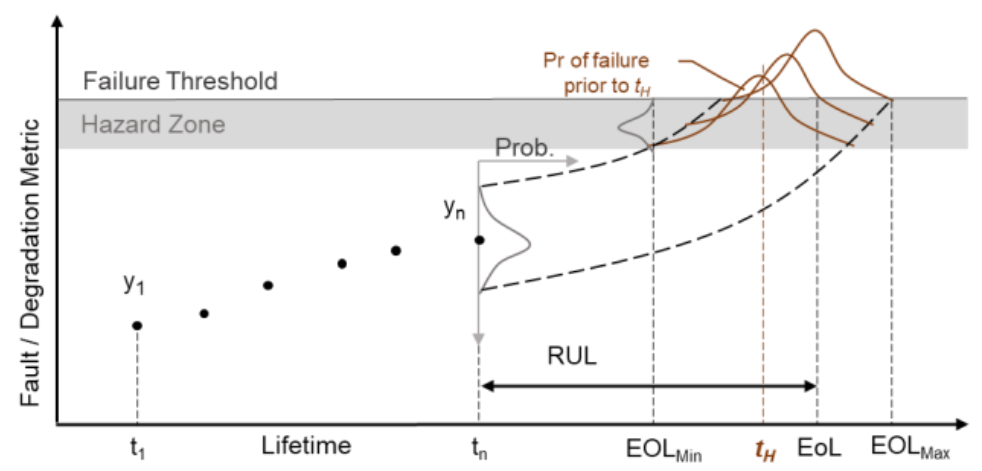

Figure 3. Prognostics based on RUL

Data driven prognostics are based on machine learning algorithms applied to degradation data collected, without making any assumption about the physics of failure or the damage accumulation model. Several machine learning techniques have been successfully applied for RUL estimation in the automotive industry, including Principle Components Analysis (Liu et al., 2016), Extreme Learning Machine, Support Vector Machines and Artificial Neural Networks (Fink et al., 2015), k-NN classifier and Bayesian filter (Mossalam et al., 2016), and random forest (Prytz et al., 2015). See Lee et al. (2014) for a wider review of examples of data driven methods for RUL prediction.

Hybrid or fusion approaches (Elattar et al., 2016) are increasingly used to address practical issues with model based approaches (e.g. where knowledge of the physics of failure or a base life model is not available to support degradation modelling) and data driven approaches (e.g. where insufficient training data is available for the model development). For example, Daigle and Goebel (2011) have illustrated the application of a particle filter approach to address issues with limited sensing data available for prognostics of a valve system.

The issues associated with the uncertainty quantification for prognostics was discussed by Sankararaman et al. (2014), in relation to four categories of uncertainty:

5. Present uncertainty - relating to the ability to estimate robustly the current state of the component based on the available sensor data;

6. Future uncertainty - which is the most significant source of uncertainty for prognostics due to the fact that the future is unknown, i.e. both the loading and the operating / environmental conditions cannot be known precisely.

7. Model uncertainty - relates to uncertainty associated with model parameters - both current and future; if model parameters have bias or are not accurate (underfitting or overfitting), then there will be discrepancies between the model predictions and actual RUL.

8. Modelling method uncertainty - if a model based architecture is used for prognostics, then even if all the other uncertainties are known (in the sense that they can be modelled probabilistically), the propagation of uncertainty through the stochastic combination of processes (e.g. as illustrated in Figure 3) yields uncertainty that needs to be addressed.

\section{PROGNOSTICS AND IVHM CHALLENGES FOR AUTOMOTIVE SYSTEMS}

While the IVHM concept has been introduced for a relatively long time (see for example Benedettini et al., 2009), there are very few mature, comprehensive implementations of IVHM systems. The challenges with prognostics and health management systems have been discussed and summarised by many authors (see for example Lee et al., 2014; Kim et al., 2017; Elattar et al., 2016, Schleigh et al., 2017). Some of these challenges, will be discussed herein from an automotive systems perspective, emphasizing the differences between different industry sectors.

\subsection{Limitation of sensing data}

A common problem across complex systems is that the "ground" parameter that is associated with the component degradation cannot be directly monitored (see for example the discussion in Doikin et al. (2018) based on a case study on an engine oil pump). This affects the effectiveness of model based prognostics approaches to estimate the state of health of the component. Alternative data driven approaches could be used, for example based on generic condition monitoring devices (vibration, temperature), that 
aim to exploit the transmission of the fault or damage progression of the component fault to neighbouring locations (as an effect of the fault or failure mode), and to detect disturbances and robustly correlated with specific component causes using machine learning algorithms. The application of such dedicated condition monitoring devices is fairly limited within automotive powertrain systems, primarily due to the cost associated with accurate and robust solutions. The justification of such solutions in other industries - like aerospace, is based on the much higher value of the product, as well as the higher safety requirements triggered by the much more severe consequences of system failure.

A common design strategy for automotive powertrain systems is to optimise the number of sensors required for functional control of the system, and use hybrid prognostics approaches for system diagnostics in relation to system functional parameters. For example, Soleimani et al. (2018) have reviewed such approaches used for automotive aftertreatement systems - in particular to deal with control failures. While such functional performance monitoring devices provide data that can be used to diagnose the state of health of physical components within the system (e.g. Doikin et al. (2018) discussed the use of oil gallery pressure monitoring to diagnose the state of health of the oil pump), the problem is that system functional monitoring sensors confound the effect of many underpinning component root causes (e.g. the oil gallery pressure is also influenced by the quality of oil, which is also subject to degradation), so it is often very difficult to derive accurate state observing variables and algorithms.

Optimising the placement of a limited number of sensors to jointly maximise the functional monitoring of the system and allow comprehensive component and system state of health assessment is an important direction for further research.

\subsection{Duty cycles and environmental uncertainty}

Compared to aerospace and nuclear industries, automotive systems are typically exposed to a much higher level of stochastic uncertainty in the duty cycles and external environment noise space. This makes both prognostics and health management decisions a lot more complex compared to other sectors, where implementation of IVHM has achieved deeper levels of maturity.

This places a greater challenge for innovation and methodology development for the integrated healthcare of automotive systems. While restricting the stochastic nature of the loads from both the user and the environment is not really possible, gathering data about duty cycles and operating conditions is increasingly feasible due to the SoS connectivity of the automotive systems. For example, from an aftertreatment system perspective, ECU data analysis can provide information about driver style and behaviour. If this is combined with current information about the environment (available through the SoS sensors) and mission (e.g. route planning and GPS data), this can generate preview information that can be used to (i) support robust differential healthcare decisions (which could mean that driver A could be allowed to continue to use the system for a longer period of time compared to driver $\mathrm{B}$, based on the mission and environment information); and (ii) implement smart adaptive predictive control of the system such that the system could achieve its current mission even if system healthcare threats are present (e.g. the propulsion system, including both the engine and the electric system in a hybrid propulsion, and the aftertreatment system can work collaboratively to meet the current target and complete the user required mission).

A challenge with implementing this strategy is that the systems will generate large amounts of data (given the multiplicative effect of the very large number of systems in operation at any one time, and the volume of data generated by the hundreds or thousands of sensors monitoring every unit at the underlying plant operating frequency) beyond the current (and very likely future) capability for transmission for offline analysis and processing. Therefore, IVHM systems like those used for aero engines, are simply not feasible for an automotive application.

It is interesting to reflect that the closest to the automotive systems paradigm in terms of prognostics is in fact the human personalised healthcare problem based on Big Data (see Viceconti et al. (2015) for an overview of big data for personalised healthcare). This conclusion is similar to that drawn by Davis (2003) in comparing reliability challenges across fields.

\subsection{System hierarchy and component data and models}

Schleigh et al. (2017) have identified that integrating digital twin technology is limited by the lack of alignment between physical and digital data driven models. This significantly affects the effectiveness of data driven models for system level healthcare, as they are unable to establish robust causal linkages across the system. While approaches to address this have been discussed, see for example Roychoudhuri et al. 
(2013) who have proposed a data and mathematical model driven computational causality framework, developing a systematic approach for integrating engineering systems analysis models with data driven models is still a fundamental challenge. This requires the integration of systems engineering models and failure analysis models that explain the causal relationships within the system or product service system (Kimita et al., 2018), with the data objects and models derived from the analysis of data collected from various sensor sources.

Figure 4 illustrates the analysis of an aftertreatment system case study across several decomposition levels of the system, identifying the complex relationships between the structural elements of the aftertreatment system, the propagation of effects from component faults to system diagnostics (only one example considered - SCR catalyst degradation), and the alignment of sensor data sources with the structure elements and functional layers of the system. This could also be represented in a network / structure matrix analysis format, as suggested by Felke et al. (2017) based on the JA6268 guidelines.

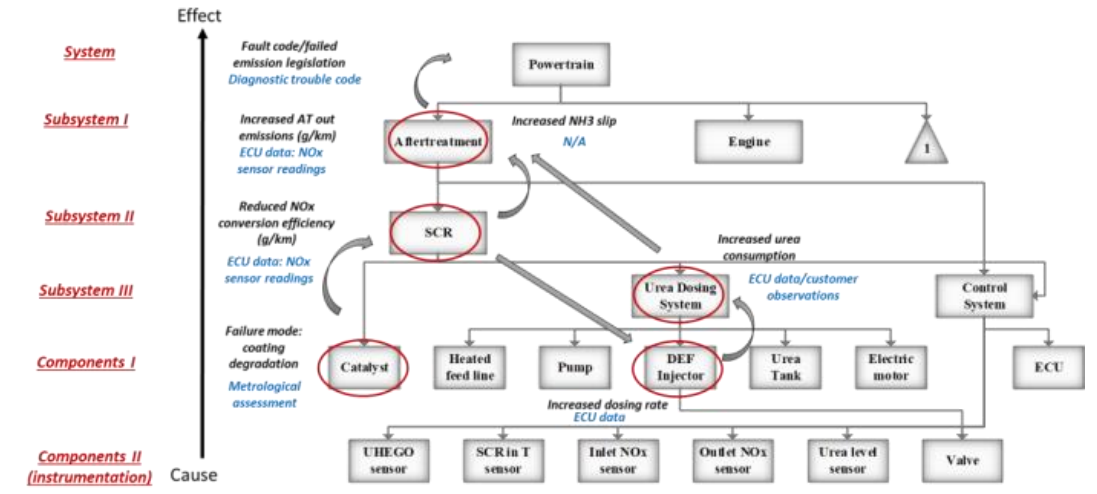

Figure 4. Aftertreatment system analysis: causal analysis and sensor data alignment

\section{AUTOMOTIVE HEALTHCARE ANALYTICS FACTORY}

Development of a comprehensive framework for integrated automotive systems healthcare in a way that addresses the prognostics and health management challenges outlined in the previous section requires: (i) an integrated life-cycle approach to data and information gathering; and (ii) systematic processing of this Big Data through descriptive, predictive and prescriptive machine learning and analytics to generate information and knowledge to underpin actionable insight. We refer to this approach as the Automotive Healthcare Analytics Factory (HAF), illustrated in Figure 5.

The distinctive feature of the HAF is that we take a holistic system lifecycle approach, which extends the current approach to digital twins through integration with Industry 4.0 lifecycle concepts. The rationale for this approach is the realisation that all system lifecycle phases provide useful data for system performance and prognostics modelling:

- Design \& Development: systems engineering and digital models and simulations of the system provide the fundamental models for function and performance that support the establishment of causal models for fault propagation, and identification of key risks to be addressed through design (including the design of the sensors and diagnostics architecture); data collected from product development testing (including both durability tests and test parts dissection) provide the basis for establishing the base life prediction models, that underpin model based prognostics.

- Manufacturing: contemporary manufacturing systems and processes operate a data rich environment that generates a digital passport for every component and subassembly; the use of this information within the manufacturing process ensures quality, whereas test data from manufacturing parts provide additional data to establish and validate the life prediction models.

- Use: conventional system healthcare frameworks, in particular data driven prognostics, rely exclusively on data collected from the use phase of the system lifecycle; this includes both offline data (such as warranty and service repair history) and online data collected from the system (diagnostics, ECU data) and from the interaction with the smart environment.

- Retirement: Dissection of retired components provide essential validation of the models that underpin prognostics.

Management and systematic processing all of this data is a Big Data challenge; collectively, this data fits the volume, velocity, variety and variability, veracity and value criteria for Big Data features (Viceconti 
et al., 2015). The Healthcare Analytics Factory systematically handles several data analytics and modelling challenges: data and model governance, including heterogeneous data quality and representation for ease of retrieval and use; machine learning systematically deployed across heterogeneous and imbalanced data sets to develop models that can be tested and validated; axiomatic categorisation to support integration of system models and data models for prognostics; statistical and stochastic modelling and simulation to derive prognostics and other probabilistic properties of the system. This is illustrated in Figure 5 in terms of the data and model driven processes (DS-DQ-DT-ML-KX-V) that underpin the inner structure of the HAF. In terms of the HAF outcomes, the prognostics feed into a healthcare decision support system, which generate prescriptive personalised action on the system. The systems models, updated with data driven insight, are fed back into the system design and development factory, updating the product knowledge based system, and informing system updates as well as future product generation.

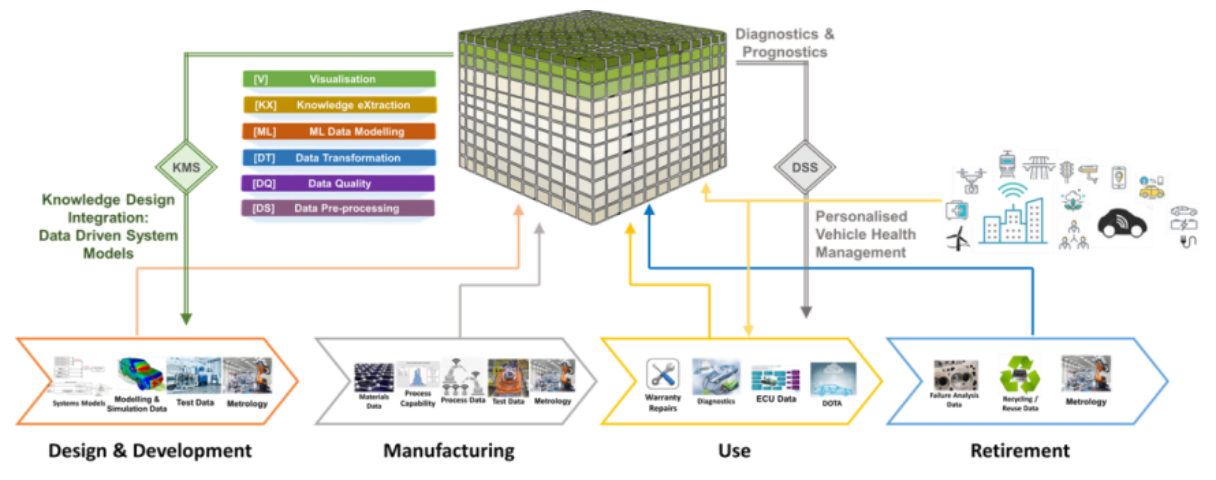

Figure 5. Automotive healthcare analytics factory

\section{CONCLUSIONS AND OUTLOOK}

Underpinned by the contemporary view of automotive systems as cyber-physical systems, characterised by increasingly open architectures and increasingly defined by their interaction with the users and the smart environment, this paper has provided a critical and up-to-date review of automotive IVHM systems. The challenges with prognostics and health management for automotive systems have been discussed in contrast with other fields - in particular aerospace and human healthcare. Given the different positions of automotive and aerospace within the volume-value quadrant, many of the IVHM solutions from aerospace are not immediately feasible for the automotive field. However, the automotive field shares much with the human healthcare field, including the fallacy of the model based only basis for prognostics (eloquently discussed by Viceconti (2015) for the human healthcare filed).

The paper has introduced a high level framework referred to as the Automotive Healthcare Analytics Factory, as a systematic, lifecycle based Industry 4.0 approach to systematically process heterogeneous Big Data sources towards prescriptive insight for personalised healthcare and knowledge integration into design. The development and implementation of the HAF is work in progress that requires substantive trans-disciplinary collaboration. Some of the case studies referred to in this paper (engine oil pump and aftertreatment system) are early test cases for the development of the integrated engineering and machine learning approach. The centrepiece of the HAF is the axiomatic categorisation framework (Byrne et al., 2018), which facilitates the association of physical entities with data objects, yielding the data driven systems engineering models that underpin the automotive digital twin.

\section{REFERENCES}

Benedetini, O., Baines, T. S., Lightfoot, H. W. and Greenough, R. M. (2009), "State-of-the-art in integrated vehicle health management”. Proc IMechE, Part G: Journal of Aerospace Eng, Vol. 223, pp. 157-170.

Byrne, T. J., Campean, F. and Neagu, D. (2018), "Towards a framework for engineering big data: An automotive systems perspective”, International Design Conference - Design 2018. http://doi.org/10.21278/idc.2018.0490

Daigle, M. and Goebel, K. (2011), “A Model Based Prognostics applied to Pneumatic Valves”, Int Jrnl of Prognostics $\&$ Health Management, Vol. 2 No. 2, pp. 1-16.

Davis, T. (2003), "Reliability improvement in automotive engineering”. In Global Vehicle Reliability - Prediction and Optimization Techniques, JE Strutt and PL Hall (Eds), PEP, London.

Doikin, A., Zadeh, E. H., Campean, F., Priest, M., Brown, M. and Sherratt, A. (2018), "Impact of duty cycle on wear progression in variable displacement vane oil pumps”, Procedia Manufacturing, Vol. 16, pp. 115-122, http://doi.org/10.1016/j.promfg.2018.10.170 
Elattar, H.M., Elminir, H.K. and Riad, A.M. (2016), "Prognostics: a Literature Review”, Complex Intell. Syst., Vol. 2, pp. 125-154, http://doi.org/10.1007/s40747-016-0019-3.

Felke, T., Holland, S., and Raviram, S. (2017), "Integration of Component Design Data for Automotive Turbocharger with Vehicle Fault Model Using JA6268 Methodology”, SAE Int. J. Passeng. Cars - Electron. Electr. Syst., Vol. 10 No. 2, 2017, http://doi.org/10.4271/2017-01-1623.

Fink, O, Zio, E., and Weidmann, U. (2015), “A classification framework for predicting components remaining useful life based on discrete event diagnostic data", IEEE Transactions on Reliability, Vol. 64 No. 3, pp. 1049-1056.

Holland, S.W. (2010), Integrated Vehicle Health management in the Auto Industry, in Smigorski, K (Editor) Health Management, IntechOpen, ISBN: 978-953-307-120-6.

Kim, N-H., An, D., and Choi, J-H. (2017), Prognostics and Health Management of Engineering Systems, Springer, http://doi.org/10.1007/978-3-319-44742-1.

Kimita, K., Sakao, T. and Shimomura, Y. (2018), "A failure analuysis method for designing highly reliable productservice systems”, Res Eng Design, Vol. 29, pp. 143-160, http://doi.org/10.1007/s00163-017-0261-8.

Kritzinger, W., Karner, M., Traar, G., Henjes, J. and Sihn, W. (2018), "Digital Twin in manufacturing: A categorical literature review and classification”, IFAC PapersOnLine, Vol. 51 No. 11, pp. 1016-1022, http://doi.org/10.1016/j.ifacol.2018.08.474

Lee, J. et al. (2014), "Prognostics and Health Management design for rotary machinery systems - Reviews, methodology and applications", Mechanical Systsems and Signal Processing, Vol. 42, pp. 314-334. http://dx.doi.org/10.1016/j.ymssp.2013.06.004

Liu, B., Yan, F., Hu, J., Turkson, R.F. and Lin, F. (2016), "Modeling and Multi-Objective Optimization of NOx Conversion Efficiency and NH3 Slip for a Diesel Engine”, Sustainability, Vol. 8 No. 5, pp. 478-491, http://dx.doi.org/10.3390/su8050478.

Mosallam, A., Medjaher, K. and Zerhouni, N. (2016), "Data-driven prognostic method based on Bayesian approaches for direct remaining useful life prediction”, Jrnl. Intel. Manuf., Vol. 27 No. 5, pp. 1037-1048.

Negri, E., Fumagalli, L. and Macchi, M. (2017), “A review of the roles of Digital Twin in CPS-based production systems”, Procedia Manufacturing, Vol. 11 No. 2017, pp. 939-948, http://dx.doi.org/10.1016/j.promfg.2017.07.198.

Prytz, R, Nowaczyk, S, Rögnvaldsson, T. and Byttner, S. (2016), "Predicting the need for vehicle compressor repairs using maintenance records and logged vehicle data", Eng Appl of Artificial Intelligence, Vol. 41, pp. 139-150.

Rosen, R., Von Wichert, G., Lo, G. and Bettenhausen, K. D. (2015), “About The Importance of Autonomy and Digital Twins for the Future of Manufacturing”, IFAC PapersOnLine, Vol. 48, pp. 567-572, http://dx.doi.org/10.1016/j.ifacol.2015.06.141.

Roychoudhury, I., Daigle, M.J., Bregon, A. and Pulido, B. (2013), “A Structural Model Decomposition Framework for System Health Management”, Proc IEEE Aerospace Conf, Big Sky, MT. http://dx.doi.org/10.1109/AERO.2013.6496975

SAE International (2018), Design \& Run-Time Information Exchange for Health-Ready Components, Surface Vehicles / Aerospace Recommended Practice JA6268 2018-04.

Sankararaman, S, Daigle, M.J. and Goebel, K. (2014), "Uncertainty Quantification in remaining Useful Life Prediction Using First Order Reliability Methods”, IEEE Trans Reliability, Vol. 63 No. 2, pp. 603-619.

Schleich, B., Anwer, N., Mathieu, L. and Wartzack, S. (2017), "Shaping the digital twin for design and production engineering”, Cirp Annals-Manufacturing Technology, Vol. 66, pp. 141-144.

Soleimani, M., Campean, F. and Neagu, D. (2018), "Reliability Challenges for Automotive Aftertreatment Systems: a State-of-the-art Perspective", Procedia Manufacturing, Vol. 16, pp. 75-82, http://dx.doi.org/10.1016/j.promfg.2018.10.174.

Viceconti, M., Hunter, P. and Hose, R. (2015), "Big Data, Big Knowledge: Big data for Personalised Healthcare", IEEE Journal of Biomedical and Health Informatics, Vol. 19 No. 4, pp. 1209-1215, http://dx.doi.org/10.1109/JBHI.2015.2406883.

Wei, Z., Rebandt, R., Start, M., Gao, L., Hamilton, J. and Luo, L. (2015), “Approaches to Achieving High Reliability and Confidence Levels with Small Test Sample Sizes”. SAE International Journal of Commercial Vehicles, Vol. 8, pp. 343-354, http://dx.doi.org/10.4271/2016-01-0269.

Weyer, S., Meyer, T., Ohmer, M., Gorecky, D. and Zuhlke, D. (2016), "Future Modelling and Simulation of CPSbased factories: an Example from the Automotive Industry”, IFAC PapersOnLine, pp. 49-31, 97-102, http://dx.doi.org/10.1016/j.ifacol.2016.12.168.

Wilmering, T. (2017), "Integrated Vehicle Health management - System of Systems Integration", SAE International, ISBN 978-0-7680-8428-3.

\section{ACKNOWLEDGMENTS}

The work presented in this paper is part of a collaborative research project on "Intelligent Personalised Powertrain Health Care", funded by Jaguar Land Rover. 